\title{
Double Retrograde Atrial Response After Radiofrequency Ablation of Typical AV Nodal Reentrant Tachycardia
}

\author{
STEVEN J. KALBFLEISCH, M.D., S. ADAM STRICKBERGER, M.D., \\ JOHN D. HUMMEL, M.D., BRIAN D. WILLIAMSON, M.D., K. CHING MAN, D.O., \\ VICKEN R. VORPERIAN, M.D., JONATHAN J. LANGBERG, M.D., \\ and FRED MORADY, M.D. \\ From the Department of Internal Medicine, Division of Cardiology, \\ University of Michigan Medical Center, Ann Arbor, Michigan
}

\begin{abstract}
Double Atrial Response. This case report describes a patient in whom a single ventricular depolarization resulted in a double atrial response and the initiation of atypical AV nodal reentrant tachycardia after successful radiofrequency ablation of typical AV nodal reentrant tachycardia using the slow pathway approach. (J Cardiovasc Electrophysiol, Vol. 4, pp. 695-701, December 1993)
\end{abstract}

atrioventricular nodal reentrant tachycardia

\section{Introduction}

A double ventricular response resulting from a single atrial depolarization conducting down dual atrioventricular (AV) nodal pathways has been previously well characterized. ${ }^{1-6}$ However, a double atrial response as a result of a single ventricular depolarization conducting across dual retrograde $\mathrm{AV}$ nodal pathways has not been well described. ${ }^{7-9}$ This case report describes a patient in whom a single ventricular depolarization resulted in a double atrial response and the initiation of atypical AV nodal reentrant tachycardia after successful radiofrequency ablation of typical AV nodal reentrant tachycardia using the slow pathway approach.

\section{Case Report}

A 24-year-old woman with recurrent paroxysmal supraventricular tachycardia was referred for electrophysiologic evaluation. During the initial electrophysiologic study, typical AV nodal reentrant tachycardia with a cycle length of $310 \mathrm{msec}$

Address for correspondence: Steven J. Kalbfleisch, M.D., Division of Cardiology, University of Michigan Medical Center, 1500 East Medical Center Drive, Ann Arbor, MI 48109-0022. Fax: 313-9367641.

Manuscript received 3 May 1993; Accepted for publication 12 July 1993. and an HA interval during tachycardia of $50 \mathrm{msec}$ was reproducibly induced in the baseline state. The tachycardia was eliminated after four applications of radiofrequency current directed at the slow pathway. Target sites were selected based on an AV electrogram ratio of $\leq 0.5$ and either a multicomponent atrial electrogram or evidence of a possible slow pathway potential during sinus rhythm. The site of attempted ablation of the slow pathway was along the tricuspid annulus at the level of the superior aspect of the coronary sinus ostium. After delivery of radiofrequency energy, sustained, typical AV nodal reentrant tachycardia could not be induced either before or during an infusion of isoproterenol. However, anterograde dual AV nodal physiology and single typical AV nodal echoes were still present.

Two months after the initial radiofrequency ablation procedure, the patient returned for an electrophysiologic evaluation because of recurrent palpitations. The $\mathrm{AH}$ interval was $80 \mathrm{msec}$ at a sinus cycle length of $580 \mathrm{msec}$. Dual AV nodal physiology was present, with anterograde fast and slow pathway effective refractory periods, at an atrial pacing drive cycle length of 500, of $290 \mathrm{msec}$ and $210 \mathrm{msec}$, respectively (Fig. 1). During an infusion of isoproterenol at a rate of $2 \mu \mathrm{g} / \mathrm{min}$, single typical AV nodal echoes with an HA interval of 50 msec could be reproducibly induced. However, sustained typical AV nodal reentrant tachycardia 


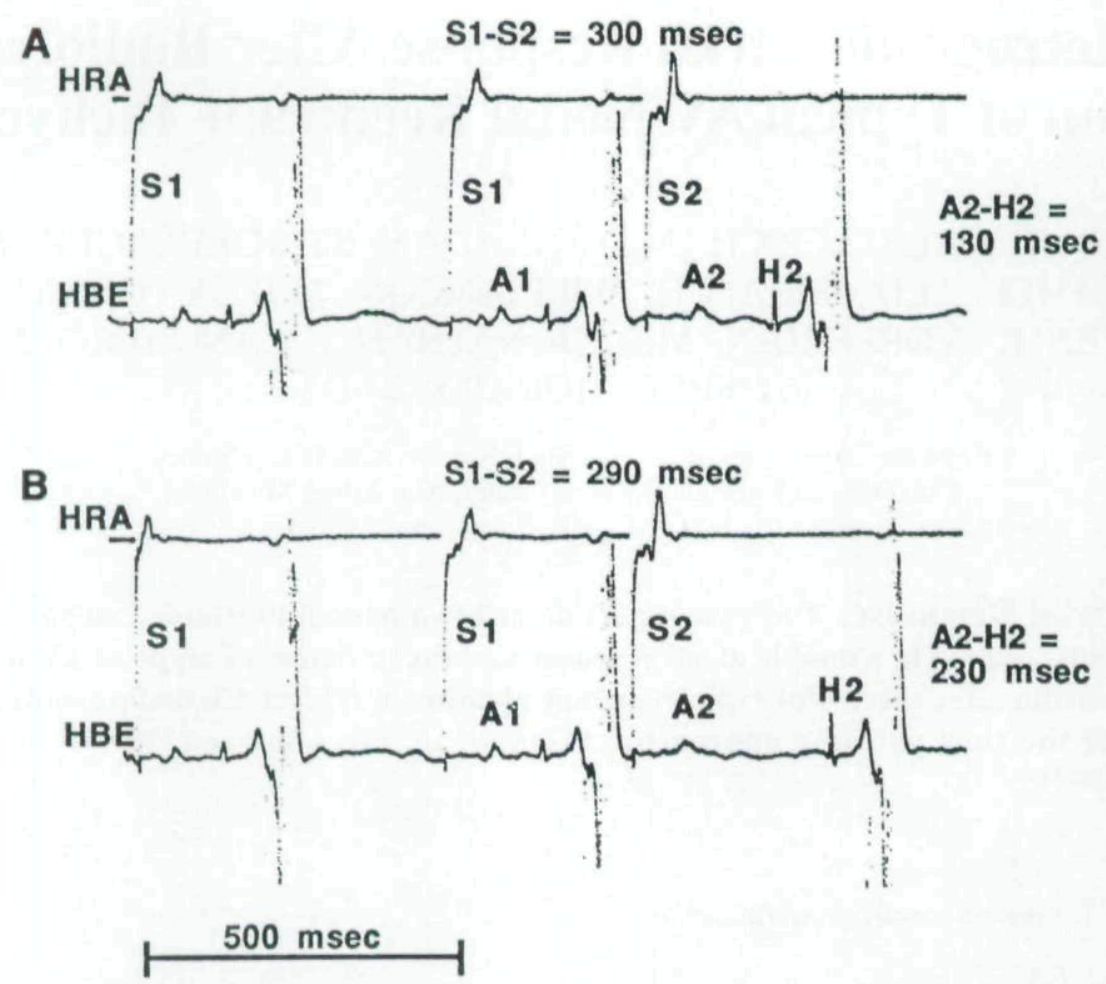

Figure 1. Antegrade dual AV node physiology. Panels $A$ and $B$ show intracardiac recordings from the high right atrium (HRA) and His-bundle electrocardiogram (HBE) recorded during atrial extrastimulation using a basic drive train cycle length (S1-S1) of $500 \mathrm{msec}$. In panel A, the extrastimulus coupling intervals (S1-S2) is $300 \mathrm{msec}$ and the A2-H2 is 130 msec. In panel B, the extrastimulus coupling interval is $290 \mathrm{msec}$ and the A2-H2 interval has jumped to 230 msec, consistent with block in the fast pathway and conduction down the slow pathway.

was not inducible either before or during the infusion of isoproterenol.

Ventricular overdrive pacing in the absence of isoproterenol revealed that ventriculoatrial conduction occurred across both a retrograde fast and slow pathway (Fig. 2A). The ventriculoatrial block cycle length of the fast pathway was $290 \mathrm{msec}$. Ventricular pacing at cycle lengths longer than the ventriculoatrial block cycle length of the fast pathway resulted in a double atrial response to the last paced ventricular depolarization and the induction of atypical AV nodal reentrant tachycardia. With ventricular pacing at cycle lengths shorter than the ventriculoatrial block cycle length of the fast pathway, the last paced ventricular depolarization often conducted across only the retrograde slow pathway, with initiation of atypical AV nodal reentrant tachycardia (Fig. 2B). The ventriculoatrial conduction time of the slow pathway was shorter when fast pathway conduction was not present (Fig. 2), possibly due to the elimination of concealed anterograde conduction in the slow pathway from the fast pathway impulse. The tachycardia cycle length was $560 \mathrm{msec}$ and the HA and $\mathrm{AH}$ intervals during tachycardia were $510 \mathrm{msec}$ and $50 \mathrm{msec}$, respectively.

During an infusion of isoproterenol at $2 \mu \mathrm{g} / \mathrm{min}$, double atrial responses were consistently elicited during ventricular extrastimulation at a ventricular basic drive train cycle length of $400 \mathrm{msec}$ and extrastimulus coupling intervals of 290 to 240 $\mathrm{msec}$, which was the ventricular effective refractory period (Fig. 3). The ventriculoatrial conduction time of the fast pathway atrial response was constant at $75 \mathrm{msec}$ and the ventriculoatrial conduction time of the slow pathway atrial response was decremental and ranged from 700 to $790 \mathrm{msec}$. With each double atrial response, atypical AV nodal reentrant tachycardia was initiated. Double atrial extrastimuli resulted in both typical and atypical $\mathrm{AV}$ node echoes and the initiation of atypical AV nodal reentrant tachycardia (Fig. 4). Both of the echoes were induced from the same paced atrial depolarization. This pattern of conduction could be consistently reproduced using an atrial basic drive cycle length of $400 \mathrm{msec}$, A1-A2 coupling 
A
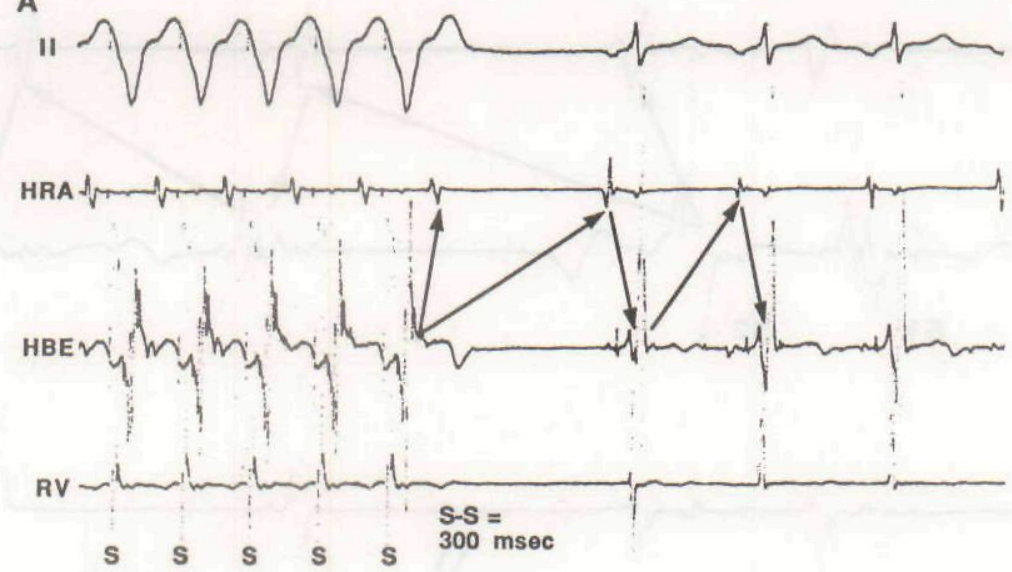

B
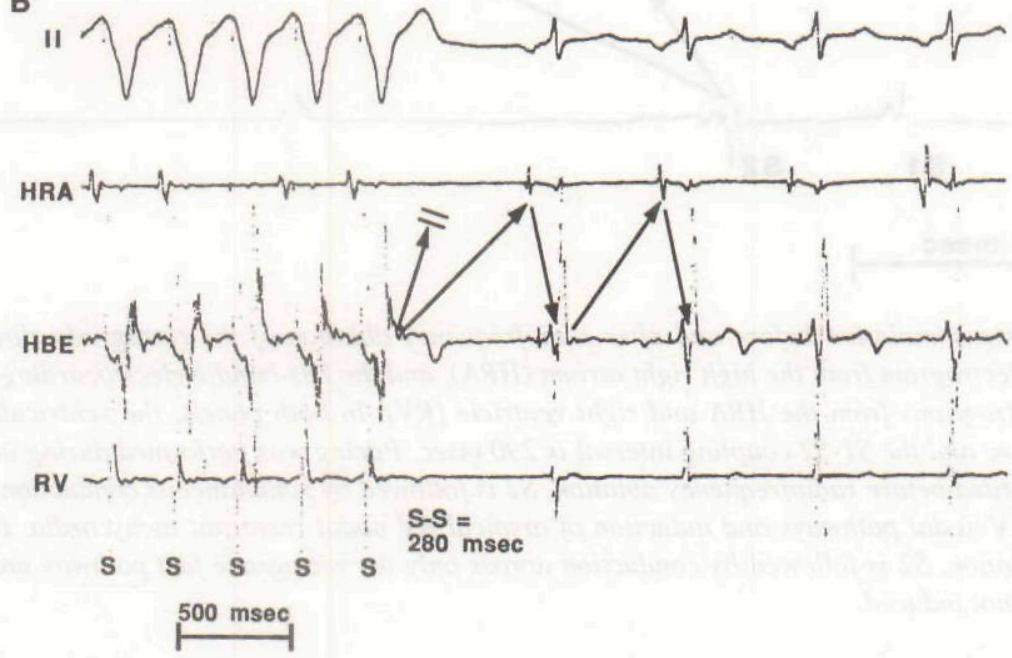

Figure 2. Retrograde fast and slow pathway conduction. Panels $A$ and B show recordings from surface lead II and intracardiac electrograms from the high right atrium (HRA), His-bundle electrocardiogram (HBE), and right ventricle (RV). Pacing stimuli are labeled $S$. In panel $A$, with ventricular overdrive pacing at a cycle length of 300 msec, the last paced beat conducts across both the retrograde fast and slow AV nodal pathways. In panel B with ventricular overdrive pacing at a cycle length of $280 \mathrm{msec}$, the last paced beat results in conduction across only the retrograde slow pathway. In both panels, the ventricular pacing resulted in the induction of atypical AV nodal reentrant tachycardia, which had a cycle length of 560 msec and HA and AH intervals of $510 \mathrm{msec}$ and $50 \mathrm{msec}$, respectively. The morphology of the $P$ wave that was due to conduction across the retrograde slow pathway was the same as the $P$ wave morphology during atypical AV nodal reentrant tachycardia.

interval of $240 \mathrm{msec}$, and A2-A3 coupling interval of $200 \mathrm{msec}$. The tachycardia had a cycle length of $440 \mathrm{msec}$ and $\mathrm{HA}$ and $\mathrm{AH}$ intervals of 400 $\mathrm{msec}$ and $40 \mathrm{msec}$, respectively, during the isoproterenol infusion.

Radiofrequency ablation was performed to eliminate the atypical AV nodal reentrant tachycardia. Bipolar recordings from the distal electrode pair of the ablation catheter were made along the tricuspid annulus between the coronary sinus ostium and the His-bundle recording position to locate the site of earliest retrograde atrial activation during tachycardia. The shortest ventriculoatrial time recorded was $230 \mathrm{msec}$ (Fig. 5). This target site, as determined in both the right anterior oblique and left anterior oblique fluoroscopic views, was approximately $1 \mathrm{~cm}$ below the His-bundle recording position and $1 \mathrm{~cm}$ superior to the site of the previous slow pathway ablation. Radiofrequency current was applied during tachycardia at a power output of 30 watts for 30 seconds. The tachycardia terminated approximately 5 seconds after the start of the radiofrequency current application. After the energy application, neither typical nor atypical AV nodal reentrant tachycardia was inducible before or during an infusion of isoproterenol. Ret- 


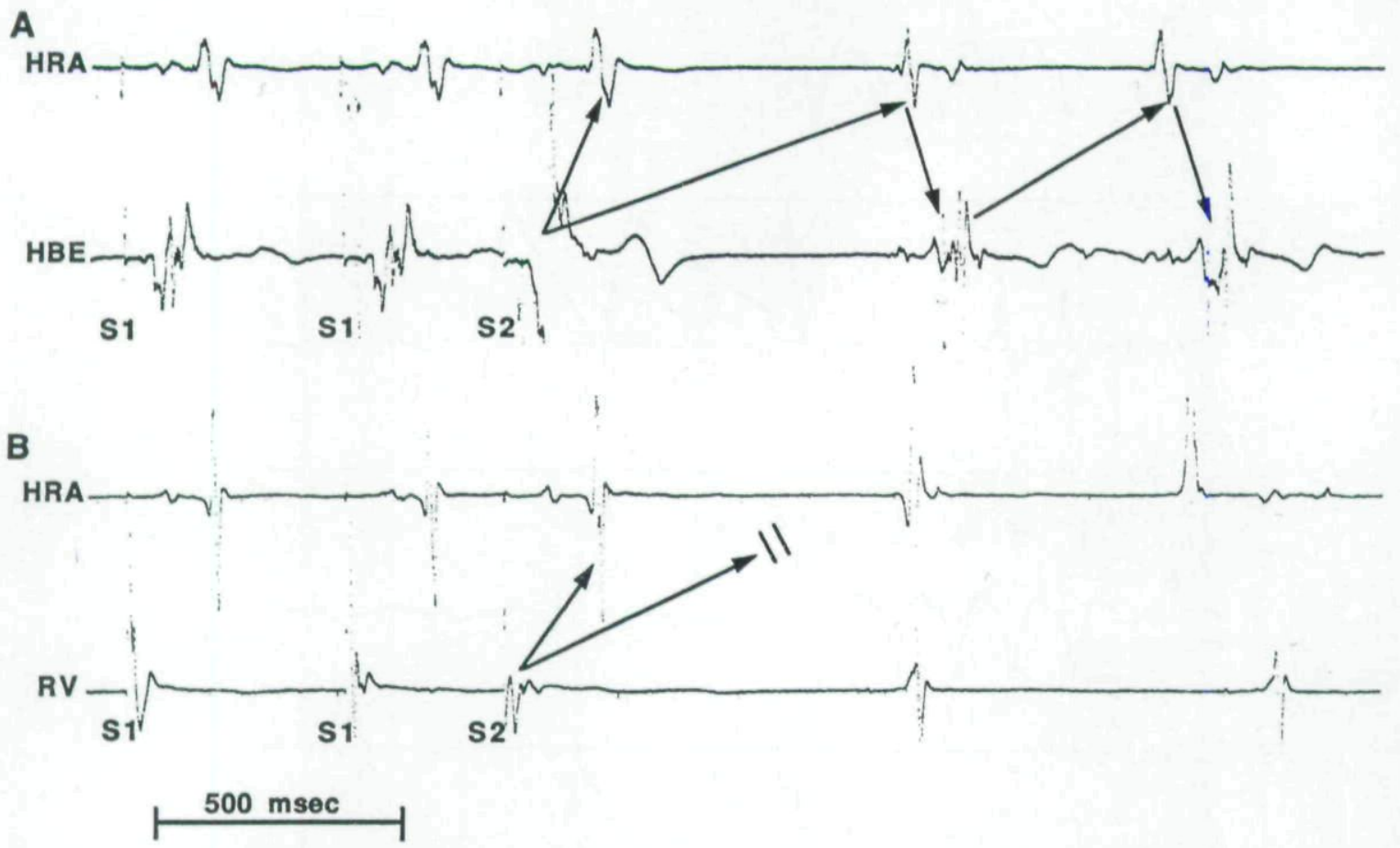

Figure 3. Ventricular extrastimulation before and after radiofrequency ablation of the retrograde slow pathway. Panel A shows an intracardiac electrogram from the high right atrium (HRA), and the His-bundle electrocardiogram (HBE). Panel B shows intracardiac electrograms from the HRA and right ventricle $(R V)$. In both panels, the ventricular basic drive cycle length (S1-S1) is $400 \mathrm{msec}$ and the S1-S2 coupling interval is $290 \mathrm{msec}$. Pacing was performed during an infusion of isoproterenol. In panel A, recorded before radiofrequency ablation, $S 2$ is followed by simultaneous conduction across both the retrograde fast and slow $A V$ nodal pathways and induction of atypical AV nodal reentrant tachycardia. In panel B, recorded after radiofrequency ablation, $S 2$ is followed by conduction across only the retrograde fast pathway and atypical AV nodal reentrant tachycardia is not induced.

rograde slow pathway conduction and the double atrial response to single ventricular extrastimuli were eliminated (Fig. 3). Anterograde dual AV nodal physiology persisted, as did the ability to induce single typical AV nodal echoes.

During a follow-up electrophysiologic test 3 weeks after the second ablation procedure, neither typical nor atypical AV nodal reentrant tachycardia was inducible before or during an infusion of isoproterenol. There was no evidence of retrograde slow pathway conduction. However, anterograde slow pathway conduction and single typical AV nodal echoes were still present.

\section{Discussion}

\section{Main Findings}

This case report describes an unusual pattern of AV nodal conduction in which a double atrial response, due to simultaneous retrograde conduction across dual AV nodal pathways, occurred in response to a single ventricular paced depolarization and resulted in the induction of atypical AV nodal reentrant tachycardia. This pattern of conduction was observed after successful radiofrequency ablation of typical AV nodal reentrant tachycardia using the slow pathway approach. After radiofrequency ablation of the retrograde slow pathway of the atypical AV nodal reentrant tachycardia, neither typical nor atypical AV nodal reentrant tachycardia could be induced. However, anterograde dual AV nodal physiology persisted and single typical AV nodal echoes could still be induced. These findings provide strong evidence for the existence of multiple slow pathways and imply that there are anatomically and functionally distinct anterograde and retrograde slow pathways.

\section{Tachycardia Mechanism}

The evidence that the induced tachycardia was atypical AV nodal reentrant tachycardia consists of the following observations: (1) the $\mathrm{AH}$ interval 

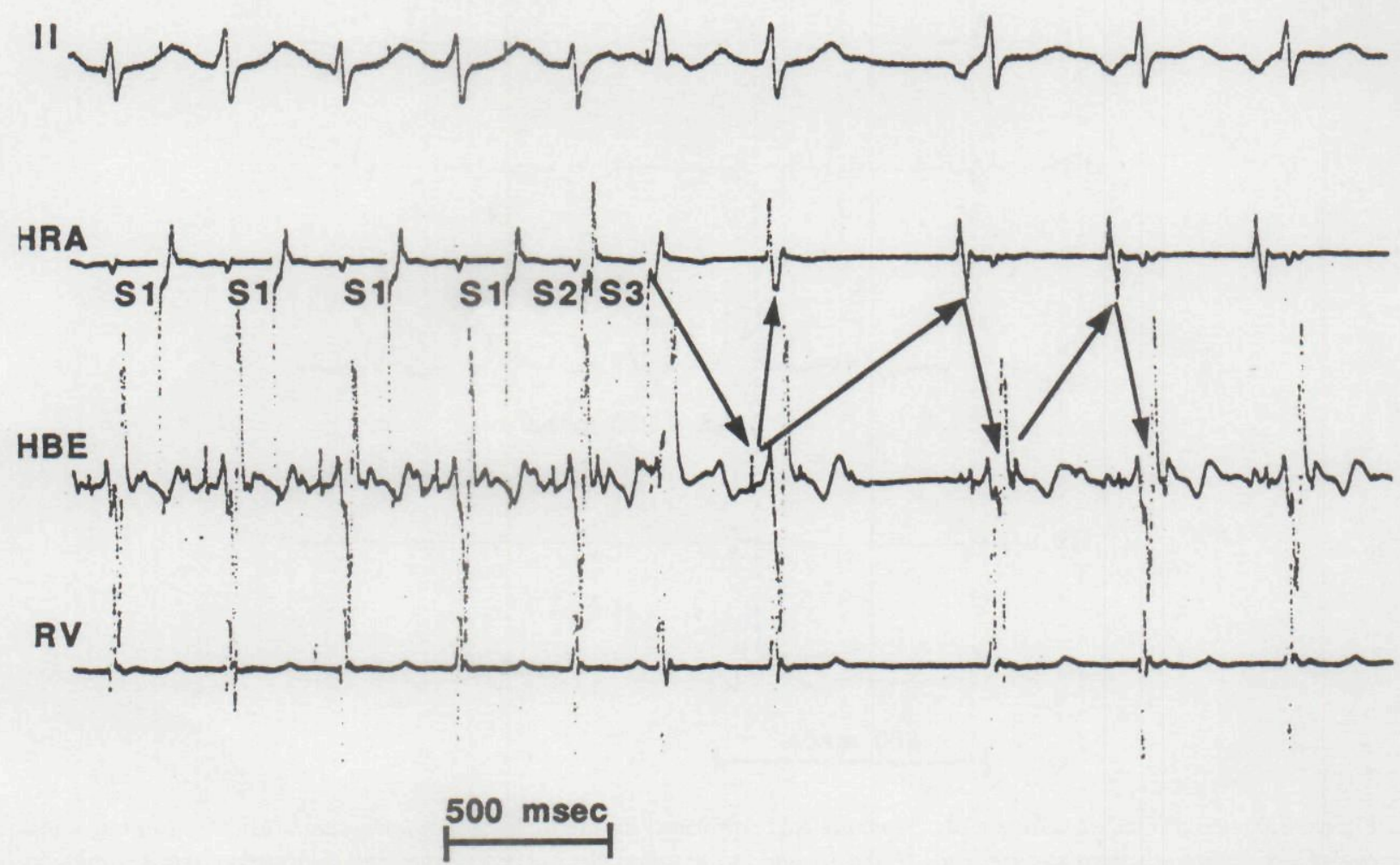

Figure 4. Double response to atrial pacing. Shown are recordings of surface lead II and intracardiac electrograms from the high right atrium (HRA), His-bundle electrocardiogram $(H B E)$, and right ventricle $(R V)$ during atrial extrastimulation. The atrial basic drive cycle length (S1-S1) is $400 \mathrm{msec}$, the S1-S2 coupling interval is 240 msec, and the S2-S3 coupling interval is 200 msec. Pacing was performed during an infusion of isoproterenol. The last atrial paced beat resulted in the induction of both a typical and atypical AV node echoes and atypical AV nodal reentrant tachycardia. The induced tachycardia had a cycle length of $440 \mathrm{msec}$ and $H A$ and $A H$ intervals of $400 \mathrm{msec}$ and $40 \mathrm{msec}$, respectively. The HA interval of the typical AV node echo was 50 msec.

during tachycardia was shorter than during sinus rhythm or atrial pacing at rates slower than the tachycardia rate; (2) the atrial electrogram could not be preexcited with ventricular extrastimuli delivered during tachycardia when the His bundle was refractory; and (3) the tachycardia could be terminated by a single ventricular depolarization that did not conduct to the atrium. The two other tachycardia types to consider in the differential diagnosis were an atrial tachycardia and an orthodromic AV reciprocating tachycardia using a slowly conducting accessory pathway. With both of these tachycardias, the $\mathrm{AH}$ interval during tachycardia should not be shorter than the $\mathrm{AH}$ interval during sinus rhythm or during rates slower than the tachycardia rate. The ability to terminate the tachycardia with a ventricular depolarization that does not depolarize the atrium rules out an atrial tachycardia and the inability to preexcite the atrium during tachycardia with a ventricular extrastimulus given when the His bundle is refractory is evidence against an AV reciprocating tachycardia utilizing an accessory pathway.

\section{Alternative Explanations}

Simultaneous conduction across retrograde fast and slow AV nodal pathways could be elicited with both ventricular and atrial pacing. Alternative explanations for the double atrial response with ventricular pacing are that the double atrial response was due to simultaneous conduction across the AV node and an accessory pathway, ${ }^{9,10}$ or that one of the atrial responses originated in the atrium. The electrophysiologic study did not reveal any evidence of an accessory pathway, but a concealed mid-septal accessory pathway with decremental conduction properties cannot be definitely ruled out. However, given the evidence that the tachycardia was atypical AV nodal reentrant tachycar- 


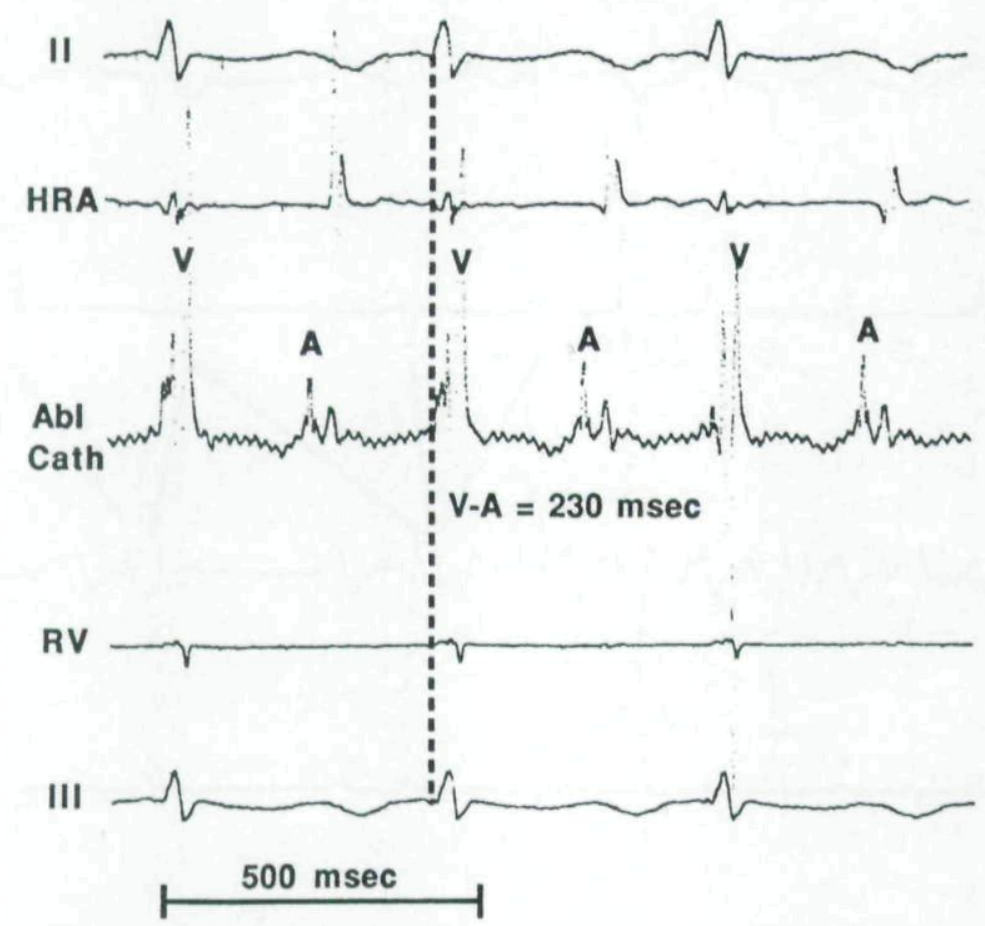

Figure 5. Successful ablation target site. Mapping was performed during tachycardia during an infusion of isoproterenol. Shown are recordings from surface leads II and III and intracardiac electrograms from high right atrium (HRA), ablation catheter $(A b l C a t h)$, and right ventricle $(R V)$. The shortest ventriculoatrial $(V-A)$ time recorded from the distal electrode pair of the ablation catheter was 230 msec. Application of radiofrequency current at this site terminated tachycardia and eliminated retrograde slow pathway conduction.

dia, this possibility seems unlikely.

The possibility that one of the two atrial responses originated in the atrium is unlikely since the response was reproducible and occurred with both ventricular overdrive pacing and with ventricular extrastimulation. The double atrial response with single atrial paced depolarization might also be explained by the occurrence of atrial premature beats or intra-atrial reentrant beats resulting from atrial pacing. The fact that the first atrial response had the same HA timing as the typical AV nodal echoes induced with single atrial extrastimuli and the fact that the second atrial response had the same $\mathrm{P}$ wave morphology as the $\mathrm{P}$ wave morphology during atypical AV nodal reentrant tachycardia make simultaneous conduction across retrograde fast and slow AV nodal pathways the most likely explanation. The other strong piece of evidence that the responses were due to simultaneous conduction across two AV nodal pathways is that after ablation of the retrograde slow pathway, the double atrial response could no longer be elicited.

\section{Prior Reports}

Two previous cases have been reported that documented the occurrence of double atrial responses to single ventricular extrastimuli as a result of conduction across both fast and slow AV nodal pathways. ${ }^{7-9}$ However, the present case is the first in which this phenomenon occurred after radiofrequency ablation of the slow pathway of typical AV nodal reentrant tachycardia and is the first to report the occurrence of double AV nodal echoes in response to a single atrial paced beat.

\section{Implications}

The occurrence of atypical AV nodal reentrant tachycardia after successful ablation of typical AV nodal reentrant tachycardia using the slow pathway approach and the fact that the site of successful ablation of the retrograde slow pathway was different than that of the anterograde slow pathway are evidence for the existence of anatomically and functionally distinct anterograde and ret- 
rograde slow pathways. The observation that the double AV nodal echoes occurred as a result of a single atrial paced beat indicates that three functionally distinct $\mathrm{AV}$ nodal pathways were present in this patient. In order for a single atrial paced beat to result in two different AV nodal echo responses, anterograde conduction must have occurred over one AV nodal pathway, followed by simultaneous retrograde conduction over two different AV nodal pathways.

The conditions that must exist for a double atrial response to a single ventricular depolarization as a result of conduction across two AV nodal pathways are: (1) a critical delay in slow pathway conduction to allow for recovery of atrial excitability; and (2) unidirectional block at the atrial insertion of the slow pathway such that the atrial depolarization from the fast pathway does not penetrate the slow pathway and block retrograde conduction. 4,6 The reason that the double atrial response did not occur before the first ablation procedure is not clear. It may be that the initial ablation attempts created the necessary unidirectional block or attenuated retrograde slow pathway conduction to a sufficient degree to achieve the necessary conduction delay for the double atrial response to occur.

\section{Conclusion}

In conclusion, this case report provides strong evidence for the existence of multiple functionally and anatomically distinct anterograde and retrograde slow pathways. The observations are helpful in understanding unusual patterns of AV nodal conduction and the existence of different types of $\mathrm{AV}$ nodal reentrant tachycardia in a given patient and explain why ablation of multiple slow pathway may be necessary in some patients to eliminate tachycardias. ${ }^{11}$

\section{References}

1. Csapo G: Paroxysmal nonreentrant tachycardias due to simultaneous conduction in dual atrioventricular nodal pathways. Am J Cardiol 1979;43:1033-1035.

2. Gomes JAC, Kang PS, Khelen G, et al: Simultaneously anterograde fast-slow atrioventricular nodal pathway conduction after procainamide. Am J Cardiol 1980;46:677-684.

3. Sutton FJ, Lee YC: Supraventricular nonreentrant tachycardia due to simultaneous conduction through dual atrioventricular nodal pathways. Am J Cardiol 1982;51:897-900.

4. Lin FC, Yeh SJ, Wu D, et al: Determinants of simultaneous fast and slow pathway conduction in patients with dual atrioventricular nodal pathways. Am Heart J 1985;109:963-969.

5. Kim SS, Lal R, Ruffy R: Paroxysmal nonreentrant supraventricular tachycardia due to simultaneous fast and slow pathway conduction in dual atrioventricular node pathways. J Am Coll Cardiol 1987;10:456-461.

6. Sakurada H, Sakamoto M, Hiyoshi Y, et al: Double ventricular responses to a single atrial depolarization in a patient with dual AV nodal pathways. PACE 1992;15:28-31.

7. Chang MS, Sung RJ, Tai TY, et al: Nadolol and supraventricular tachycardia: An electrophysiologic study. J Am Coll Cardiol 1983;2:894-903.

8. Matsuhisa M, Shimomura K, Ohe T, et al: Double atrial and double ventricular responses during slowfast fast-slow atrioventricular nodal reentrant tachycardia. PACE 1989;12:1381-1386.

9. Lin FC, Yeh SJ, Wu D: Double atrial responses to a single ventricular impulse due to simultaneous conduction via two retrograde pathways. J Am Coll Cardiol 1985;5:168-175.

10. Miles WM, Klein LS, Minardo JD, et al: Two retrograde atrial responses from one ventricular complex in the permanent form of junctional reciprocating tachycardia. Am J Cardiol 1987;59:1004-1006.

11. Jackman WM, Beckman KJ, McClellans JH, et al: Treatment of supraventricular tachycardia due to atrioventricular nodal reentry by radiofrequency catheter ablation of slow-pathway conduction. N Engl J Med 1992;327:313-318. 
Copyright of Journal of Cardiovascular Electrophysiology is the property of Blackwell Publishing Limited and its content may not be copied or emailed to multiple sites or posted to a listserv without the copyright holder's express written permission. However, users may print, download, or email articles for individual use. 